\title{
Uma análise quantitativa e qualitativa da superestrutura narrativa no Comprometimento Cognitivo Leve e na doença do Alzheimer
}

\author{
A quantitative and qualitative analysis of narrative superstructure in Mild Cognitive Impairment and \\ Alzheimer's disease
}

\section{Gislaine Machado Jerônimo Lilian Cristine Hübner}

Pontifícia Universidade Católica do Rio Grande do Sul - PUCRS - Porto Alegre - Rio Grande do Sul - Brasil Resumo: Narrativas orais fazem parte do cotidiano da população idosa. Desse modo,
torna-se relevante analisar como idosos em um processo de envelhecimento típico e
atípico constroem suas histórias. Participaram deste estudo 64 idosos com baixa
escolaridade, os quais foram divididos em três grupos: idosos neurologicamente
saudáveis, idosos com Comprometimento Cognitivo Leve e idosos com doença de
Alzheimer leve. Realizou-se uma testagem da memória de trabalho, assim como uma
avaliação linguística da produção de narrativa oral, baseada em instrumento pictórico
com sete figuras (The Dog Story, Le Boeuf, 1976; Hübner et al., 2019). Para avaliação
da superestrutura narrativa foi utilizado o Modelo de Adam (1987, 2008). Os
resultados mostraram diferença significativa entre os grupos clínicos e o grupo
controle tanto na criação da superestrutura da história quanto no desempenho do teste
de memória de trabalho. Houve uma correlação positiva entre o desempenho da
memória trabalho e o desempenho na construção da narrativa, evidenciando, assim,
uma relação estreita entre ambos os componentes da cognição.

Palavras-chave: superestrutura narrativa, memória de trabalho, Comprometimento Cognitivo Leve, doença de Alzheimer.

\begin{abstract}
Oral narratives take part of daily life of the elderly population. Therefore, the analysis of how elders in typical and atypical aging build their stories is relevant. Sixtyfour elderly people with low schooling participated in this study, divided into three groups: neurologically healthy elderly, elderly with Mild Cognitive Impairment and elderly with mild Alzheimer's disease. Working memory testing was carried out, as well as a linguistic assessment of an oral narrative production, based on a pictorial instrument with seven scenes (The Dog Story, by Le Boeuf, 1976; Hübner et al., 2019). The evaluation of the narrative superstructure was conducted according to the model proposed by Adam $(1987,2008)$. The results showed a significant difference between the clinical groups and the control group both in the creation of the story superstructure and in the performance in the working memory test. There was a positive correlation between working memory performance and the performance in the narrative construction, reflecting a close relationship between both components of cognition.
\end{abstract}

Keywords: narrative superstructure, working memory, Mild Cognitive Impairment. Alzheimer's disease. 


\section{Introdução}

No Brasil há um crescimento contínuo da população idosa, acarretando o aumento de doenças relacionadas à idade, dentre elas, o Comprometimento Cognitivo Leve (CCL) e a doença de Alzheimer (DA) (GOMES, TERRA, 2015; TOLEDO, 2018; JERÔNIMO, 2018). O CCL configura-se como um declínio cognitivo associado à idade, um estágio de transição entre 0 envelhecimento sadio e um processo demencial (ALBERT et al., 2011), enquanto a DA se caracteriza como uma doença neurodegenerativa progressiva (McKHANN et al., 2011). Visando à qualidade de vida, nestes casos, a literatura cada vez mais tem enfatizado a importância de atividades de intervenção não farmacológicas, com destaque especial ao treino cognitivo (KOCHHANN, WILSON, 2019).

Afora os componentes cognitivos costumeiramente investigados para diagnóstico de CCL e DA, como as memórias, a atenção, as funções executivas, uma investigação mais aprofundada da linguagem pode oferecer dados valiosos para 0 diagnóstico complementar das doenças em questão e subsídio para o treino cognitivo, uma vez que a linguagem representa um dos elementos cognitivos mais relevantes para a funcionalidade diária do idoso (VERNA, HOWARD, 2012). Ademais, alterações da linguagem podem estar presentes desde o início de um quadro de declínio cognitivo ou processo demencial (HÜBNER et al, 2019). No entanto, a maioria das pesquisas publicadas na área trata de prejuízos linguísticos somente no nível da palavra ou frase, sendo pouco frequentes os estudos discursivos que investigam, por exemplo, a produção de narrativas orais no CCL e na DA, em especial em populações de baixa escolaridade.

Os parâmetros internacionais são de estudos com CCL e DA de alta escolaridade, dado que reforça a importância de estudos nacionais com baixa escolaridade voltados ao envelhecimento, tanto sadio quanto atípico. Desse modo, o desenvolvimento de estudos em centros internacionais especializados em envelhecimento propicia uma oportunidade ímpar para o avanço de nossos estudos no Brasil. Em 2014, a autora principal deste estudo obteve bolsa de doutorado sanduíche pela CAPES para desenvolver pesquisa junto ao Centre de recherche de I'Institut universitaire de gériatrie de Montréal (CRIUGM), em Montreal no Canadá. Sua pesquisa foi desenvolvida sob coordenação Professor Dr. Yves Joanette, Fonoaudiólogo, Diretor do CRIUGM na época e professor da Escola de Medicina da Université de Montréal. Esta escolha se deu devido à parceria entre o grupo de parceira do professor e o grupo de pesquisa (Grupo de Pesquisa em Neurolinguística e Psicolinguística - GENP), coordenado pela Professora Lilian Cristine Hübner, do qual a primeira autora participa. O Professor Joanette é um estudioso renomado na área dos estudos da linguagem e sua ligação com demais funções cognitivas no envelhecimento sadio e atípico, em especial nas demências. O CRIUGM, instalado ao lado de um hospital geriátrico, agrega várias equipes multidisciplinares com pesquisas sobre envelhecimento, cujas discussões e experiências compartilhadas tornam o ambiente extremamente rico para todos os interlocutores. Este artigo, que apresenta parte da pesquisa realizada, tem o objetivo de investigar as características da superestrutura narrativa produzida oralmente por idosos com CCL, idosos com DA em comparação à produção de idosos neurologicamente saudáveis, bem como verificar os escores dos grupos em um teste de memória de trabalho, correlacionando-os aos de linguagem.

\section{Delineamento metodológico}

Este estudo foi transversal, observacional e contemporâneo realizado com aprovação do Comitê de Ética em Pesquisa da PUCRS, sob o número de parecer 560.073, no dia 06 de março de 2014, registro CAAE 21006913.0.0000.5336.

\subsection{Participantes}

Os participantes foram selecionados por conveniência, divididos em três grupos: idosos 
saudáveis com baixa escolaridade e dois grupos clínicos diagnosticados, respectivamente, com CCL amnéstico e provável DA leve.

Dentre os critérios de inclusão, estão idade (entre 60 e 79 anos), escolaridade (entre 2 e 8 anos de ensino formal para grupos de baixa escolaridade e acima de 9 anos para o grupo saudável de alta escolaridade) assim como grau de demência e gravidade da doença, no caso do grupo com DA e CCL, sendo que todos os pacientes deveriam ter comprometimento de memória.

Dentre os critérios de exclusão, relacionam-se história de alcoolismo, de doença neurológica (exceto CCL e DA), depressão não tratada, problemas relacionados à visão ou à audição não corrigidos e escolaridade abaixo de 2 anos de ensino formal. No caso de participantes com DA, foram excluídos os que obtiverem escores no Clinical Dementia Rating (CDR) superiores a 1.

Aplicou-se igualmente um questionário sobre aspectos gerais de saúde.

O diagnóstico de CCL e provável DA foi realizado por uma equipe multidisciplinar de um hospital de referência da cidade de Porto Alegre, composta por neurologistas, psiquiatras, psicólogos e fonoaudiólogos.

Critérios para o diagnóstico de CCL foram: a) queixa de prejuízos cognitivos por parte do paciente ou de algum familiar, ou por observação médica; b) alteração em um ou mais domínios da cognição e c) preservação funcional, isto é, manutenção da autonomia nas atividades de vida diária, conforme recomendações do Grupo de Trabalho do National Institute on Aging (NIA) e Alzheimer's Association (AA) (ALBERT et al., 2011). A avaliação cognitiva foi realizada por meio da aplicação do Mini Exame do Estado Mental (MEEM), versão adaptada para o Português Brasileiro (CHAVES, IZQUIERDO, 1992), e pela aplicação do Addenbrooke's Cognitive Examination (ACE-R) (MIOSHI et al., 2006). A funcionalidade do paciente foi verificada por meio dos escores do CDR (MORRIS, 1993; MONTAÑO, RAMOS, 2005). A avaliação do humor se deu por meio da verificação de critérios diagnósticos do CID-
10 e DSM-IV para transtornos de humor e risco de suicídio, com o auxílio da Mini Entrevista Internacional de Neuropsiquiatria - Português Versão 6.0 (M.I.N.I. Plus) (SHEEHAN et al., 2010) e aplicação do GDS (YESAVAGE et al., 1983). Complementou-se o diagnóstico com exames de sangue (hemograma, eletrólitos, TSH, creatinina, ureia, TGO, TGP, VDRL, Vitamina B12, Ácido fólico, anti-HIVe ECG) e exames de neuroimagem (Tomografia ou, preferencialmente, Ressonância Magnética cerebral).

Os critérios para o diagnóstico de provável DA foram: a) queixa por parte do paciente ou de algum familiar, ou observação médica; b) alteração em, no mínimo, dois domínios da cognição e c) comprometimento na funcionalidade. A aferição dos critérios diagnósticos para demência se deu igualmente com Base no CID-10 e Diagnostic and Statistical Manual of Mental Disorders (DSM-IV). A avaliação de humor, avaliação cognitiva e os exames clínicos para DA foram os mesmos empregados para diagnóstico de CCL.

\subsection{Instrumento para avaliação da memória do trabalho}

Após a triagem, os participantes foram avaliados pelo teste neuropsicológico Ordenamento de Ascendente de Dígitos (subteste do NEUPSILIN, de FONSECA et al., 2009), o qual avalia a memória de trabalho. Neste teste o participante deve ordenar sequências numéricas, que estão agrupadas em 10 blocos de 2 a 6 dígitos cada.

\subsection{Instrumento de avaliação da linguagem}

Para avaliação do componente linguístico, foi realizada uma testagem de produção oral de texto, com o emprego da tarefa da Bateria de Avaliação da Linguagem no Envelhecimento (BALE) (HÜBNER et al, 2019). A tarefa solicitada ao participante foi construir uma narrativa oral, com base em uma sequência de sete figuras em preto e branco, com base na História do Cachorro (LE BOUEF, 1976) (Fig. 1). A versão da História do Cachorro publicada na 
BALE (HÜBNER et al., 2019) apresenta pequenas adaptações às cenas de modo a atualizá-las e adaptá-las ao nosso contexto cultural, mantendo-se o roteiro e a quantidade de cenas da história base.

Figura 1: Instrumento linguístico de coleta de dados

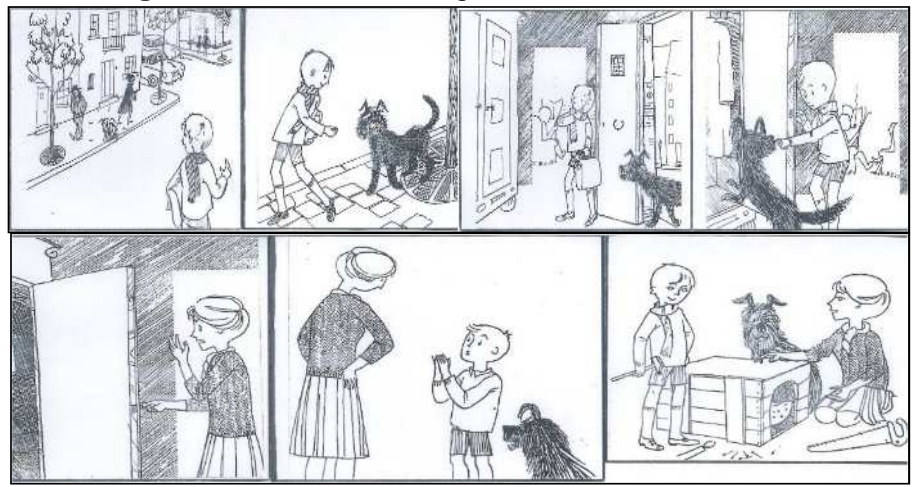

Fonte: LE BOUEF (1976).

\subsection{Procedimento para coleta de dados}

Os participantes do estudo foram individualmente convidados a contar uma história com base na sequência de figuras, apresentadas em linha, na ordem da história. A instrução dada foi: "Vou lhe mostrar uma história com figuras. Cada figura é um momento da história, que tem início, meio e fim. Vou pedir que o (a) senhor (a) olhe bem as figuras e tente entender a história. Então, vou Ihe pedir que me conte essa história como se fosse contar para um amigo/amiga. (Esperar) Está pronto (a)? Podemos começar?" A tarefa é gravada e transcrita para posterior análise.

Vislumbrando minimizar os efeitos de memória, as ilustrações ficam em frente ao participante durante o teste. Não há tempo limite para produção e a única interferência do examinador, ao longo do teste, é de encorajamento ao participante, caso ele não demonstre iniciativa na continuidade da história.

\subsection{Análise linguística}

A superestrutura narrativa foi avaliada conforme o modelo proposto por Adam (1987, 2008), descrito no Quadro 2. As análises intra e intergrupo foram feitas de forma quali/quantitativa, com foco na análise qualitativa. Juízes especialistas fizeram um pré-julgamento dos elementos que poderiam compor cada item, a fim de auxiliar na análise subsequente.

Quadro 1: Estágios da superestrutura narrativa (com base em Adam 1987, 2008)

\begin{tabular}{|l|l|}
\hline $\begin{array}{l}\text { Situação } \\
\text { inicial } \\
\text { (Pn1) }\end{array}$ & $\begin{array}{l}\text { Momento em que se definem as } \\
\text { situações de espaço, tempo e } \\
\text { características das personagens. }\end{array}$ \\
\hline $\begin{array}{l}\text { Nó } \\
\text { desencadeador } \\
\text { (Pn2) }\end{array}$ & $\begin{array}{l}\text { Ocorre após a situação inicial, por } \\
\text { meio de uma ação que visa a } \\
\text { modificar o estado inicial da } \\
\text { narrativa propriamente dita. }\end{array}$ \\
\hline $\begin{array}{l}\text { Re-ação ou } \\
\text { (Pn3) }\end{array}$ & $\begin{array}{l}\text { Re-ação: refere-se ao momento } \\
\text { que transforma a nova situação } \\
\text { provocada pela complicação; } \\
\text { avaliação: momento que indica as } \\
\text { reações das personagens. }\end{array}$ \\
\hline $\begin{array}{l}\text { Desenlace } \\
\text { (Pn4) }\end{array}$ & $\begin{array}{l}\text { Estabelecimento de um novo } \\
\text { estado, diferente do inicial da } \\
\text { história. }\end{array}$ \\
\hline $\begin{array}{l}\text { Situação final } \\
\text { (Pn5) }\end{array}$ & Fechamento da história. \\
\hline
\end{tabular}

Fonte: Adam (1987, 2008)

\subsection{Construção do texto base}

Anterior à análise de dados, dez juízes especialistas na área de linguagem, entre eles psicolinguistas, fonoaudiólogos e lógicos, realizaram a tarefa de produção narrativa com base na sequência de figuras. Doze macroproposições foram obtidas na produção dos juízes (apresentadas no Apêndice 1) e serviram de base para a análise da coerência global do texto, bem como para a classificação da estrutura narrativa.

\section{Resultados}

Considerando idade e escolaridade, houve homogeneidade entre os grupos analisados, conforme mostrou a ANOVA. 
Tabela 1: Características sociodemográficas dos grupos (idade, escolaridade)

\begin{tabular}{lcccl}
\hline & $\mathbf{C}$ & $\mathbf{C C L}$ & $\mathbf{D A}$ & COMPARAÇÕES $\boldsymbol{p}$ \\
& $\mathbf{N}=$ & $\mathbf{N =}$ & $\mathbf{N}=$ & \\
& $\mathbf{3 4}$ & $\mathbf{1 5}$ & $\mathbf{1 5}$ & \\
\hline \multirow{4}{*}{ Idade } & $\mathrm{M}$ & $\mathrm{M}$ & $\mathrm{M}$ & \\
& $(\mathrm{DP})$ & $(\mathrm{DP})$ & $(\mathrm{DP})$ & \\
\cline { 2 - 5 } Escolaridade & 69,85 & 68,13 & 71,60 & $\mathrm{C}=\mathrm{CCL}=\mathrm{DA}$ \\
& $(5,34)$ & $(5,39)$ & $(6,85)$ & $0,265^{1}$ \\
& 4,94 & 3,87 & 4,27 & $\mathrm{C}=\mathrm{CCL}=\mathrm{DA}$ \\
& $(1,85)$ & $(1,35)$ & $(1,75)$ & $0,115^{1}$
\end{tabular}

Legenda: $\mathbf{N}=$ número de participantes; $\mathbf{M}=$ média; $\mathbf{D P}=$ desvio padrão; $\mathbf{C}=$ controle; $\mathbf{C C L}=$ Comprometimento Cognitivo Leve; DA = doença de Alzheimer. Nota: ${ }^{*} p \leq 0,05$ indica diferença entre os grupos; ${ }^{1}$ (ANOVA).

No teste de Ordenamento Ascendente de Dígitos os grupos mostraram diferenças significativas. Os grupos CCL e C, bem como DA e C diferenciaram-se. Não houve diferença entre os grupos clínicos.

Tabela 2: Comparação entre o desempenho dos grupos com CCL e DA, em relação ao grupo $C$, no teste de Dígitos

\begin{tabular}{|c|c|c|c|c|c|}
\hline & $\begin{array}{c}C \\
34\end{array}$ & $\begin{array}{c}C C L \\
11\end{array}$ & $\begin{array}{l}\text { DA } \\
13\end{array}$ & & \\
\hline & $\begin{array}{c}\mathrm{M} \\
(\mathrm{DP})\end{array}$ & ${ }_{(\mathrm{DP})}{ }^{\mathrm{M}}$ & $\begin{array}{c}\mathrm{M} \\
(\mathrm{DP})\end{array}$ & Comparações & $P$ \\
\hline $\begin{array}{l}\text { Ordenament } \\
\text { o } \\
\text { Ascendente } \\
\text { de Dígitos }\end{array}$ & $\begin{array}{r}3,88 \\
(1,83)\end{array}$ & $\begin{array}{r}2,45 \\
(1,21)\end{array}$ & $\begin{array}{r}1,77 \\
(1,64)\end{array}$ & $\begin{array}{l}\mathrm{C}>\mathrm{CCL} \\
\mathrm{C}>\mathrm{DA} \\
\mathrm{CCL}>\mathrm{DA}\end{array}$ & 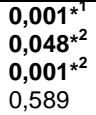 \\
\hline
\end{tabular}

Legenda: $\mathbf{N}=$ número de participantes; $\mathbf{M}=$ média; $\mathbf{D P}=$ desvio padrão; $\mathbf{C}=$ Controle; $\mathbf{C C L}=$ Comprometimento Cognitivo Leve; DA = doença de Alzheimer; $\mathbf{M}$ (média); DP (desvio padrão) Nota: ${ }^{*} \mathrm{p} \leq 0,05$ é considerado significante; ${ }^{1}$ (ANOVA); ${ }^{2}$ Análise post-hoc teste Tukey HSD

A superestrutura narrativa teve correlação positiva com o teste de Ordenamentos Ascendente de Dígitos para os grupos clínicos CCL e DA.

Tabela 3: Correlação entre os escores de memória de trabalho e análise
Os dados de correlação mostraram que quanto mais altos os escores obtidos pelos grupos clínicos no teste de Ordenamentos Ascendente de Dígitos, que afere memória de trabalho, mais altos os escores na superestrutura narrativa.

Os resultados da análise quantitativa da estrutura narrativa mostraram diferença estatística entre os grupos. Tal diferença se deu entre os grupos com CCL e DA, em relação ao grupo $C$, sem que houvesse distinção entre os dois grupos clínicos entre si (ver Tabela 4).

Tabela 4: Comparação entre o desempenho dos grupos com CCL e DA, em relação ao grupo $\mathrm{C}$, na análise da superestrutura narrativa

\begin{tabular}{|c|c|c|c|c|c|}
\hline & $\begin{array}{c}\mathrm{C} \\
\mathbf{3 4} \\
\mathrm{M} \\
(\mathrm{DP}) \\
\end{array}$ & $\begin{array}{c}\mathrm{CCL} \\
15 \\
\mathrm{M} \\
(\mathrm{DP})\end{array}$ & $\begin{array}{r}\text { DA } \\
15 \\
M \\
(D P) \\
\end{array}$ & $\begin{array}{l}\text { Comparaçõe } \\
\text { s }\end{array}$ & $p$ \\
\hline $\begin{array}{l}\text { SUPERESTRUT. } \\
\text { NARRATIVA } \\
\text { TOTAL }\end{array}$ & $\begin{array}{l}3,68 \\
(1,14)\end{array}$ & $\begin{array}{l}1,53 \\
(1,18)\end{array}$ & $\begin{array}{l}1,47 \\
(1,35)\end{array}$ & $\begin{array}{l}\mathrm{C}>\mathrm{CCL} \\
\mathrm{C}>\mathrm{DA} \\
\mathrm{CCL}>\mathrm{DA}\end{array}$ & $\begin{array}{l}<0,001^{{ }^{1}} \\
<0,001^{{ }^{2}} \\
<0,001^{{ }^{2}} \\
0,987\end{array}$ \\
\hline
\end{tabular}

Legenda: $\mathbf{N}=$ número de participantes; $\mathbf{M}=$ média; $\mathbf{D P}=$ desvio padrão; $\mathbf{C}=$ Controle; $\mathbf{C C L}=$ Comprometimento Cognitivo Leve; DA = doença de Alzheimer; Nota: ${ }^{*} p \leq 0,05$ é considerado significante; ${ }^{1}$ (ANOVA); ${ }^{2}$ Análise post-hoc teste de Tukey HSD

A fim de complementar a análise quantitativa, foi realizada uma análise qualitativa da $\mathrm{SN}$, para verificar em qual parte da $\mathrm{SN}$ a diferença entre os grupos foi maior.

A Tabela 5 apresenta a comparação entre os grupos e os percentuais de participantes em cada parte da SN.

\section{Superestrutura Narrativa}

\begin{tabular}{lc}
\hline $\begin{array}{l}\text { Grupos Clínicos CCL e DA ( } \mathbf{n}=\mathbf{3 0}) \\
\text { Ordenamentos Ascendente de Dígitos (ordem } \\
\text { inversa) }\end{array}$ &, $431^{\star}$ \\
\hline $\begin{array}{l}\text { Grupo de idosos saudáveis com baixa escolaridade ( } \mathbf{n}=34) \\
\text { Ordenamento Ascendente de Dígitos (ordem inversa) }\end{array}$ &, 027 \\
\hline
\end{tabular}

Nota: *Correlação significativa $p<0,05$; Correlação de Pearson. 
Tabela 5: Comparação entre o desempenho dos grupos com CCL e DA, em relação ao grupo $C$, na análise qualitativa da superestrutura narrativa

\begin{tabular}{llll}
\hline & \multicolumn{1}{c}{$\mathbf{C}$} & \multicolumn{1}{c}{$\mathbf{C C L}$} & \multicolumn{1}{c}{$\mathbf{D A}$} \\
\cline { 2 - 4 } & $\mathbf{N}$ & $\mathbf{N}$ & $\mathbf{N}$ \\
& $\%$ & $\%$ & $\%$ \\
\cline { 2 - 4 } n0 & 0 & 1 & 3 \\
& 0 & 6,7 & 20 \\
Pn1 & 2 & 9 & 7 \\
& 5,9 & $\mathbf{6 0 , 0}$ & $\mathbf{4 7 , 6}$ \\
Pn2 & 3 & 2 & 1 \\
& 8,8 & 13,3 & 6,7 \\
Pn3 & 8 & 1 & 2 \\
& 23,5 & 6,7 & 13,3 \\
Pn4 & 12 & 1 & 2 \\
& $\mathbf{3 5 , 3}$ & 6,7 & 13,3 \\
Pn5 & 9 & 1 & 0 \\
& 26,5 & 6,7 & 0 \\
Total & 34 & 15 & 15 \\
& 100 & 100 & 100 \\
\hline
\end{tabular}

Legenda: $\mathbf{N}=$ número de participantes; $\mathbf{C}=$ Controle; $\mathbf{C C L}=$ Comprometimento Cognitivo Leve; $\mathbf{D A}=$ doença de Alzheimer; (n0) - não situou espaço, tempo e características das personagens; Situação inicial (Pn1): momento em que se definem as situações de espaço, tempo e características das personagens; Nó desencadeador (Pn2): ocorre após a situação inicial, por meio de uma ação que visa modificar o estado inicial da narrativa propriamente dita. Re-ação ou Avaliação (Pn3): re-ação culmina no momento que transforma a nova situação provocada pela complicação; avaliação: momento que indica as reações das personagens. Desenlace (Pn4): estabelecimento de um novo estado, diferente do inicial da história. Situação final (Pn5): fechamento da história.

No grupo C, a maior concentração de participantes foi na Pn4, isto é, $(35,3 \%)$ chegaram até o desenlace; (26,5\%) atingiram a Pn5 - situação final, tendo relatado uma história com todos os elementos estruturais da narrativa, de acordo com Adam (1987, 2008); (8,8\%) ficaram na Pn2 e (23,5\%) na Pn3; apenas $(5,9 \%)$ dos participantes do grupo $C$ atingiram a Pn1, momento em que se estabelece o cenário da narrativa.

Já os grupos de idosos com CCL e DA tiveram maior concentração de participantes na Pn1 ( $\mathrm{CCL}=$ $60,0 \%$ ) e (DA $=46,7 \%)$. No grupo com $C C L$, apenas $(6,7 \%)$ dos participantes chegaram na Pn5 - situação final, enquanto nenhum participante do grupo com DA conseguiu finalizar a história. Os percentuais de desempenho demostram que a maior parte dos idosos de ambos os grupos com CCL e DA fixou-se na situação inicial, parte mais elementar da narrativa em que são realizadas mais descrições, não evoluindo para as ações posteriores. Em tais grupos houve ainda a ocorrência de participantes que nem chegaram na Pn1, pois, ou eles contaram uma história pessoal com base em algum elemento das figuras, ou utilizaram estratégias para suprir as dificuldades e não iniciaram a contação da história solicitada; neste caso, classificamos estes textos em um novo parâmetro - n0 ( $\mathrm{n}$ zero).

\section{Discussão}

Referente ao Teste de Dígitos Ascendente (ordem inversa), comparando idosos com CCL, DA e controles, Stormoen et al. (2014) e Jacinto et al. (2014) não encontraram diferenças entre os grupos CCL e DA no teste de Dígitos (ordem inversa). Jacinto e colegas (2014) investigaram o desempenho de idosos brasileiros com baixa escolaridade. Os resultados obtidos por estes pesquisadores na diferenciação de grupos clínicos com CCL e DA, em relação ao grupo $\mathrm{C}$, vêm ao encontro dos nossos achados. O declínio dos grupos clínicos pode estar associado à dificuldade de manipulação e monitoramento da informação por um curto período de tempo (DENIS, CABEZA, 2008).

A memória de trabalho, de acordo com o modelo de Baddeley et al. (2011), é gerenciada pelo executivo central, que controla o nível atencional durante uma tarefa específica e garante que as estratégias utilizadas se relacionem ao seu objetivo. As dificuldades que o grupo com $\mathrm{CCL}$ e, sobretudo o grupo com DA, demonstraram no teste de memória de trabalho sugerem, portanto, que este gerenciador, o executivo central, pode não estar monitorando adequadamente, em tais grupos, os níveis atencionais e as estratégias necessárias à resolução da tarefa.

A memória de trabalho está envolvida em muitos aspectos da linguagem, relacionados a operações simultâneas (MANSUR et al,. 2005). Na DA a memória de trabalho é mais prejudicada (HUNTLEY, HOWAR, 2009). Porém, quanto maior a capacidade do idoso com CCL e DA em manter as 
informações na memória de trabalho, melhor o desempenho em contar uma narrativa oral.

A análise dos resultados obtidos pelos grupos no quesito estrutura da narrativa mostrou que os grupos CCL e DA não atingiram o que Adam (1987) chama de sentido configuracional pragmático da história, pois eles, de modo geral, relataram acontecimentos que não foram significativos para atingir a moral. Nas narrativas produzidas, nem sempre parece claro o objetivo, o enredo da narrativa em especial na DA, como ilustra o segmento abaixo:

\begin{abstract}
"aqui eu acho que o meninozinho queria conversar (com o agradar o cachorrinho, né?) ... e aqui ele ia saindo... o cachorro parece que tava meio brabo com ele ... ele fez assim e tá indo atrás dele ... aqui ele deu uma coisinha pra ele comer ó é ... e aqui (vá) essa moça tentou abrir a porta acho que era do roupeiro de alguma coisa né... - -eu reparei ali- - ...é, e aqui este menino (venceu) na moto que chegou nela... né? ... na moça ó ... ahhh, e o cachorrinho ficou do lado ... e ali - -olha que bonito- - ... a moça ficou ajoelhada... o menino perto e o cachorrinho junto... - -olha que lindo né? uhum ... hmm que bonito. mais algum detalhe? alguma coisa moça? (viu) direitinho o que eu errei?".
\end{abstract}

No exemplo apresentado, o participante traz elementos do cenário, identifica o menino, o cachorro, percebe que o cachorro está indo atrás do menino, porém na sequência não indica para onde, nem o que irão fazer. A moça surge na história, o participante identifica o roupeiro, mas não estabelece relação entre os fatos, não percebe a complicação e introduz falas que não têm ligação com as figuras, como é o caso da expressão "da moto". A história não é finalizada, há apenas uma descrição final, em que a "moça" (mãe), o cachorro e o menino aparecem juntos. Adam (1987) chama a atenção para o fato de que os significados devem emergir da história. Com base na história citada, não é possível depreenderem-se os significados, uma vez que não há a passagem do estado inicial para o final.

Conforme o exemplo, parece que os idosos com DA têm dificuldades de perceber que uma figura está relacionada com a outra, formando uma história. Eles demostram entender as figuras de forma isolada, como se fossem autônomas umas das outras, não estabelecendo uma relação hierárquica entre elas. $O$ mesmo ocorreu no grupo com DA investigado por Cardebat e colegas (1993). Além das dificuldades de encadeamento das partes da história, há um comprometimento iconográfico na DA (CARDEBAT et al., 1993). Tais déficits são perceptíveis quando observamos 0 encadeamento estabelecido em relação às ações das personagens, pois muitos dos idosos com DA parecem não ter entendido que havia um número limitado de personagens, que se repetiam e mantinham-se na história, realizando diferentes ações. Eles identificaram várias personagens ao longo das figuras, como mostra o exemplo a seguir:

“isso é uma pracinha - - eu acho - -... uma
criança ( ) um cachorrinho... outra criança
(não é?)... - - não sei o que que é isso aqui...
é uma porta né? - -... um cachorro um
cachorro... outra criança... (uma) guria... mais
dois... esse bichinho - - aqui eu não sei o que
que é...- - o gurizinho tá conversando com o
cachorrinho né... tá dando comidinha pro
cachorrinho... e aqui... aqui o cachorrinho tá
atendendo ela aqui também né... aqui a
guriazinha tá brincando com o cachorrinho...
aqui tá abrindo a porta ó... aqui tá essa
senhora aqui...".

$\mathrm{Na}$ primeira figura da história há diferentes pessoas na rua e o participante as identifica parcialmente. Seria o menino ou uma criança (possível protagonista, que aparece em todas as cenas), um cachorro, dois adultos (um homem e uma mulher) próximos ao cachorro e uma mulher com uma criança na esquina, o que totaliza cinco pessoas e um cachorro. O participante identifica três crianças ou quatro, ao invés de duas, pois não se sabe se "uma guria" está explicando que uma das crianças é do sexo feminino ou se é outra personagem que ele identificou, embora pela figura não seja possível identificar o sexo da criança que está na esquina. Algumas das pessoas que aparecem na primeira figura fazem parte apenas do cenário e não aparecerão posteriormente, mas o participante, mesmo depois de ter tido um tempo para olhar todas as figuras e entender a história antes de iniciar a produção oral, escolheu identificar todas as pessoas que estavam na figura inicial. Na sequência do texto, o participante identificou uma guriazinha e uma 
senhora, sem perceber que seriam a mesma personagem, possivelmente a mãe ou a avó do menino, personagem protagonista.

Este idoso, então, informou partes secundárias e de menor relevância ao todo da história, vindo ao encontro do postulado por Cardebat, Démonet e Doyon (1993). Os autores defendem que pessoas com DA se fixam em detalhes das figuras; o que igualmente explica a maior concentração de idosos com DA na Pn1.

Nossos resultados corroboraram os encontrados por Chapman et al. (1995), Ska e Duong (2005), Lira (2014) e Cardebat, Démonet e Doyon (1993), pois assim como neste estudo, todos os autores citados encontraram diferenças significativas entre o desempenho dos grupos de idosos com DA e os respectivos grupos controles, no que tange à SN baseada em figuras. Não foram encontrados estudos com DA que tenham evidenciado resultados contrários aos aqui evidenciados - a partir da adoção de estímulo formado por sequência de figuras.

Cardebat Démonet e Doyon (1993), além de investigarem o desempenho de idosos com DA comparado ao de controles saudáveis, compararam idosos com DA com idosos afásicos. Os autores analisaram a presença ou não de complicação na história e verificaram que quase todos os idosos do grupo controle mencionaram a complicação, porém nenhum idoso com DA conseguiu mencioná-la. Na comparação entre os afásicos e os idosos com DA, os afásicos tiveram mais dificuldades com a produção narrativa, mais prejuízos sintáticos e com o léxico do que as pessoas com DA, o que pode ter interferido na produção oral deles. Já os idosos com DA apresentaram mais dificuldades com a seleção dos elementos da história, isto é, com a elaboração da macroestrutura narrativa, fixando-se nos detalhes das figuras.

Lira (2014), assim como nós e Cardebat, Démonet e Doyon (1993), fez uso da mesma sequência narrativa, a História do Cachorro (LE BOUEF, 1976). A autora não analisou a história observando a mesma sequência proposta por Adam. Ska e Duong (2005) analisaram a organização da narrativa por meio de dois instrumentos pictóricos, figura única e sequência de figuras, identificando dificuldades na DA para ambos os instrumentos. Chapman et al. (1995) investigaram a produção narrativa oral de três grupos: DA, controle e idosos longevos com mais de 80 anos e verificaram que os grupos controle e de idosos longevos tiveram melhor desempenho do que o grupo com DA.

O grupo com DA do presente estudo, em uma análise de todos os elementos da $\mathrm{SN}$, conforme Adam (1987, 2008), poucas vezes relatou a complicação da história, apresentando déficits em todas as partes da narrativa, vindo ao encontro dos achados de Toledo et al. (2018), em que o grupo com DA produziu narrativas com menor informatividade. Lira et al. (2018) igualmente encontraram resultados semelhantes aos nossos. A observância à SN parece, desse modo, refletir uma dificuldade própria da DA, pois os participantes do presente estudo tiveram acesso às figuras durante todo o tempo do teste, as quais foram apresentadas na ordem correta dos acontecimentos, a fim de facilitar a organização das ideias e diminuir a carga das memórias episódica e de trabalho, dificuldades estas que não forma percebidas no grupo controle.

Entretanto, as dificuldades com a SN parecem não ser exclusivas à DA, pois elas foram igualmente evidenciadas no CCL. Tais déficits, desse modo, já podem ser identificados em fases anteriores à DA. Drummond et al. (2015) corroboram esta hipótese. No presente estudo, assim como em Drummond et al. (2015), não houve diferenças significativas entre o desempenho do grupo CCL e DA na SN, embora tais grupos tenham se diferenciado do grupo controle nas médias de acertos. Qualitativamente podemos verificar que o grupo com DA inseriu várias personagens na história, o que não ocorreu com o grupo com CCL. O grupo com CCL, porém, inseriu alguns elementos não pertinentes à história. A SN, proposta por Adam (1987, 2008), é, portanto, um importante marcador linguístico na diferenciação de idoso saudáveis de grupos clínicos com CCL e DA. 


\section{Considerações finais}

Os grupos clínicos apresentaram dificuldades com a superestrutura narrativa, estabelecendo o cenário, mas evoluindo pouco para os acontecimentos posteriores. O grupo com DA não chegou à situação final da história, diferentemente do grupo com $\mathrm{CCL}$ em que alguns dos participantes finalizaram a narrativa. Prejuízos neste sentido foram associadas a uma memória de trabalho comprometida e a dificuldades em identificar e reportar a superestrutura da narrativa.

O relato de narrativas é importante porque é ecologicamente válido e por fazer parte da vida diária de idosos. A cadeia de uma narrativa, com sua estrutura já bem delimitada por diferentes modelos, como o de Adam, parece ser uma ferramenta útil para identificar a preservação ou declínios na habilidade de narrar.

Os dados desta pesquisa podem trazer contribuições para o diagnóstico complementar e precoce de $C C L$ e DA, bem como para a criação de programas de treino cognitivo para idosos em um processo de envelhecimento típico e atípico.

\section{Referências}

ADAM, Jean-Michel.Types de Séquences Textuelles Élémentaires. (Alexânia Rippol et all, 1992, Trad.) In: Pratiques. n.56, dec, 1987.

ADAM, Jean-Michel. A linguística textual: introdução à análise textual dos discursos. São Paulo: Cortez, 2008.

ALBERT, Marilyn S. et al. The diagnosis of mild cognitive impairment due to Alzheimer'sdisease: Recomendations from the National Institute on AgingAlzheimer's Association workgroups on diagnostic guideline for Alzheimer's disease. Alzheimer's \& Dementia, v.7, p. 270-279, 2011.

ALMEIDA, Osvaldo P.; ALMEIDA, Shirley A. Short version of the geriatric depression scale: a study of their validity for the diagnosis of a major depressive episode according to ICD-10 and DSM-IV. International Journal of Geriatric Psychiatry, v. 14, p.858-865, 1999.

BADDELEY, Alan.; ANDERSON, Michael.; EYSENCK, Michael. Memória. Porto Alegre: Artes Médicas, 2011.
BRANDÃO, Lenisa. Perfil discursivo de pessoas com Doença de Alzheimer. Porto Alegre: UFRGS, 2005. Tese (Tese em Psicologia), Faculdade de Psicologia, Universidade do Rio Grande do Sul, 2005, $185 f$.

CARDEBAT, D.; DEMONET, J.F.; DOYON, B. Narrative discourse in dementia. In: Brownell $\mathrm{HH}$, Joanette $\mathrm{Y}$ (Eds.). Narrative discourse in neurologically impaired and normal aging adults. San Diego: Singular; 1993. p. 317-22.

CÉSAR, Karolina. Estudo da prevalência de comprometimentocognitivo leve na cidade de Tremembé, estado de São Paulo. Tese (Tese em Medicina), Faculdade de Medicina, Universidade de São Paulo, 2014, $137 f$.

CHAPMAN, Sandra Bond et al. Discourse in early Alzheimer's disease versus normal advanced aging. American Journal of Speech-Language Pathology, v. 4, p. 124-129, 1995.

CHAVES, Márcia.,; IZQUIERDO, Ivan. Differential diagnosis between dementia and depression: A study of efficiency increment. Acta Neurologica Scandinavica, v. 85, p. 378-382, 1992.

DENNIS, Nancy A.; CABEZA, Roberto. Neuroimaging of healty cognitive aging. In: CRAIK, Fergus.; SALTHOUSE, Timothy. The handbook of aging and cognition (3rd Ed). New York: Psychology Press Taylor \& Francis Grou, 2008, p. 1-54.

DRUMMOND, Cláudia et al. Deficits in narrative discourse elicited by visual stimuli are already presented in patients with mild cognitive impairment. Frontiers in Aging Neuroscience, v.7, p. 1-11, 2015.

FONSECA, Rochele. Paz.; SALLES, Jerusa.; PARENTE, Maria. Alice. Mattos. Pimenta. Instrumento de Avaliação Neuropsicológica Breve Neupsilin. São Paulo: Vetor Editora, 2009.

FROTA, Norberto Anízio Ferreira et al. Critérios para o diagnóstico de doença de Alzheimer. Dementia \& Neuropsychologia, v. 5, p. 5-10, 2011.

HÜBNER, Lilian. Cristine.; LOUREIRO, Fernanda.; SMIDARLE, Anderson.; TESSARO, Bruna. ; SOARES, Ellen.; JERÔNIMO, Gislaine. et al. Bateria de Avaliação de Linguagem no Envelhecimento (BALE). In: ZIMMERMANN, NICOLE; DELAERE, François; FONSECA, Rochele. Paz. (Org.). Tarefas para Avaliação Neuropsicológica para idosos: avaliação de memória episódica, percepção, linguagem e componentes executivos para adultos. 1ed.Rio de Janeiro: Memnon, 2019, v. 3, p. 188-221.

HUNTLEY, J. D.; HOWARD, R. J. Working memory in early Alzheimer's disease: a neuropsychological review. International Journal of Geriatric Psychiatry: A journal of the psychiatry of late life and allied sciences, v. 25, n. 2, p. 121-132, 2010.

JACINTO, Alessandro Ferrari et al. Suggested instruments for general practioners in countries with 
low schooling to screen for impairment in the elderly. International Psychogeriatrics, p. 1-5, 2014.

JERÔNIMO, Gislaine Machado. Envelhecimento sadio, Comprometimento Cognitivo Leve e doença de Alzheimer: um estudo das estratégias comunicativas na narrativa oral. Letras de Hoje, v. 53, n. 1, p. 177186, 2018.

KOCHHANN, Renata; WILSON, Maximiliano. Introduction: Special issue on neuropsychology of aging. Psychology \& Neuroscience, v. 12, n. 2, p. 141, 2019.

LAKS, Jerson et al. Mini-Mental State Examination norms in a community-dwelling sample of elderly with low schooling in Brazil / Normas do Mini-Exame do Estado Mental para uma amostra de idosos com baixa escolaridade residentes na comunidade no Brasil. Cad Saúde Pública, 23:315-319, 2007.

LE BOUEF, Christine. Raconte - 55 historiettes en images. Paris: L'Ecole, 1976.

LIRA, Juliana. Onofre. Análise dos aspectos macrolinguísticos e desenvolvimento de um índice de avaliação do discurso oral em pacientes com doença de Alzheimer. São Paulo: Tese não publicada: São Paulo, UNIFESP, 2014. Tese não publicada (Doutorado em Ciências). Faculdade de Medicina, Universidade Federal de São Paulo, 2014. $138 f$.

LIRA, Juliana. Onofre et al. Evaluation of macrolinguistic aspects of the oral discourse in patients with Alzheimer's disease. International psychogeriatrics, p. 1-11, 2018.

MCKHANN, Guy M. et al. The diagnosis of dementia due to Alzheimer's disease: Recommendations from the National Institute on Aging-Alzheimer's Associations workgroups on diagnostic guidelines for Alzheimer's disease. Alzheimer's \& Dementia, v. 7, p. 263-269, 2011.
MIOSHI, Eneida et al. The Addenbrooke's Cognitive Examination Revised (ACE-R): a brief cognitive test battery for dementia screening. International Journal of Geriatric Psychiatry: A journal of the psychiatry of late life and allied sciences, v. 21, n. 11, p. 10781085, 2006.

MACEDO MONTAÑO, Maria Beatriz M.; RAMOS, Luiz Roberto. Validade da versão em português da Clinical Dementia Rating. Revista de Saúde Pública, v.39, n.6, p. 912-917, 2005.

MORRIS, John C. The Clinical Dementia Rating (CDR): Current version and scoring rules. Neurology, v.43, 2412-2414, 1993.

SHEEHAN, D. $\mathrm{V}$ et al. The Mini - International Neuropsychiatric Interview (M.I.N.I.): Mini Entrevista Neuropsiquiátrica Internacional: Portuguese for Brazil translation for DSM - IV to CID-10, Version 6.0.0. Mapi Research Institute, 2010.

SKA, Bernadette., DUONG, Anh. Communication, discourse and dementia. Psychologie \& NeuroPsychiatrie du Vieillissement, v. 2, p. 125-33, 2005.

STORMOEN, Sara et al. Cognitive predictors of medical decision-making capacity in mild cognitive Impairment and Alzheimer's disease. International Journal of Geriatric Psychiatry, v. 12, p. 1304-1311, 2014.

TOLEDO, Cíntia Matsuda et al. Analysis of macrolinguistic aspects of narratives from individuals with Alzheimer's disease, mild cognitive impairment, and no cognitive impairment. Alzheimer's \& Dementia: Diagnosis, Assessment \& Disease Monitoring, v. 10, p. 31-40, 2018.

VERMA, Malvika; HOWARD, R. J. Semantic memory and language dysfunction in early Alzheimer's disease: a review. International Journal of Geriatric Psychiatry, v. 27, p. 1209-1217, 2012.

\section{COMO CITAR ESSE ARTIGO}

JERÔNIMO, Gislaine Machado; HÜBNER, Lilian Cristine. UMA ANÁLISE QUANTITATIVA E QUALITATIVA DA SUPERESTRUTURA NARRATIVA NO DECLÍNIO COGNITIVO LEVE E NA DOENÇA DE ALZHEIMER. Signo, Santa Cruz do Sul, v. 44, n. 81, jan. 2020. ISSN 1982-2014. Disponível em: $<$ https://online.unisc.br/seer/index.php/signo/article/view/13992>. Acesso em: doi: https://doi.org/10.17058/signo.v44i81.13992. 



\begin{tabular}{|c|c|c|c|c|}
\hline & & $\begin{array}{c}\text { (interpretação mais plausível com base nas } \\
\text { histórias dos juízes) }\end{array}$ & $\begin{array}{c}\text { Proposição 2 (continuação da 1) } \\
\text { (interpretação mais plausível com } \\
\text { base nas histórias dos juízes) }\end{array}$ & $\begin{array}{l}\text { Indicativos das } \\
\text { figuras } \\
\text { (descrição dos } \\
\text { objetos presentes e } \\
\text { gestos das } \\
\text { personagens) }\end{array}$ \\
\hline \multirow[t]{2}{*}{ Pn1 } & $\begin{array}{l}\text { CENA } \\
1\end{array}$ & $\begin{array}{l}\text { o menino está (na rua/ andando na } \\
\text { rua/caminhando na rua) } \\
\text { Aceitável: } \\
\text { (menino/guri/garoto) } \\
\text { (tempo verbal presente [ contínuo] ou } \\
\text { passado) } \\
\text { (história com discurso direto e indireto) } \\
\text { (voz ativa e voz passiva) } \\
\text { ponto) }\end{array}$ & $\begin{array}{l}\text { [ele] vê/encontra um cachorro } \\
\text { está olhando para um cachorro } \\
\text { Aceitável: } \\
\text { (cachorro/cachorrinho) } \\
\text { (1 ponto) }\end{array}$ & $\begin{array}{l}\text { Há duas pessoas, uma } \\
\text { mulher e uma criança, } \\
\text { um cachorro e um } \\
\text { menino na rua. } \\
\text { Há dois carros na } \\
\text { esquina. }\end{array}$ \\
\hline & $\begin{array}{l}\text { CENA } \\
2\end{array}$ & $\begin{array}{l}\text { o menino chama o cachorrinho (se aproxima do } \\
\text { cachorrinho/atrai o cachorrinho) [para sua casa] } \\
\text { Aceitável: } \\
\text { (ele/menino) } \\
\text { ponto) }\end{array}$ & & $\begin{array}{l}\text { Há um menino e um } \\
\text { cachorro na rua. } \\
\text { O menino faz gestos } \\
\text { para o cachorro. }\end{array}$ \\
\hline Pn2 & $\begin{array}{l}\text { CENA } \\
3\end{array}$ & $\begin{array}{l}\text { o menino chega/entra em casa } \\
\text { O menino deixa o cachorro entrar } \\
\text { [levou o cachorro pra casa] } \\
\text { ponto) }\end{array}$ & $\begin{array}{l}\text { faz sinal para o cachorro fazer silêncio } \\
\text { (ficar quieto/ não fazer barulho) } \\
\text { [pede silêncio para o cachorro] } \\
\text { (1 ponto) }\end{array}$ & $\begin{array}{l}\text { Um menino e um } \\
\text { cachorro entram em } \\
\text { um local. } \\
\text { O menino está com a } \\
\text { mão na boca, fazendo } \\
\text { gesto para o cachorro. }\end{array}$ \\
\hline Pn3 & $\begin{array}{l}\text { CENA } \\
4\end{array}$ & $\begin{array}{l}\text { o menino esconde/coloca o cachorro dentro do } \\
\text { armário } \\
\text { aponta/ fala para o cachorro ficar ali [no } \\
\text { armário] } \\
\text { Aceitável: } \\
\text { (armário/roupeiro/guarda-roupa) } \\
\text { ponto) }\end{array}$ & & $\begin{array}{l}\text { Um menino e um } \\
\text { cachorro estão em } \\
\text { frente um armário, o } \\
\text { menino está } \\
\text { apontando o dedo para } \\
\text { o cachorro entrar no } \\
\text { local/ armário. }\end{array}$ \\
\hline Pn3 & $\begin{array}{l}\text { CENA } \\
5\end{array}$ & $\begin{array}{l}\text { a mãe abre o armário } \\
\text { Aceitável: } \\
\text { (armário/roupeiro/guarda-roupa) } \\
\text { ponto) }\end{array}$ & $\begin{array}{l}\text { [a mãe] encontra /acha/descobre/vê) } \\
\text { o cachorro } \\
\text { Aceitável: } \\
\text { (encontra/acha/descobre/vê) } \\
\text { (1 ponto) }\end{array}$ & $\begin{array}{l}\text { A mulher (mãe) abre a } \\
\text { porta do armário, } \\
\text { coloca a mão para } \\
\text { cima, abre a boca e vê } \\
\text { o cachorro. }\end{array}$ \\
\hline Pn3 & $\begin{array}{l}\text { CENA } \\
6\end{array}$ & $\begin{array}{l}\text { a mãe (tira/pede ) (satisfação/explicação) } \\
\text { a mãe questiona o menino } \\
\text { a mãe briga com o menino } \\
\text { Aceitável: } \\
\text { (tirar/pedir) } \\
\text { (satisfação/explicação) } \\
\text { ponto) }\end{array}$ & $\begin{array}{l}\text { o menino (pede/insisti/implora) para } \\
\text { ficar com o cachorro } \\
\text { o menino diz que (quer/gostaria de) } \\
\text { ficar com o cachorro } \\
\text { o menino pede desculpa [por colocar o } \\
\text { cachorro no armário,/por levar o } \\
\text { cachorro para casa) } \\
\text { Aceitável: } \\
\text { (pede/insisti/implora) } \\
\text { (quer/gostaria de) }\end{array}$ & $\begin{array}{l}\text { A mulher (mãe) e o } \\
\text { filho estão } \\
\text { conversando. Ela está } \\
\text { com as mãos na } \\
\text { cintura e ele com as } \\
\text { mãos jutas em forma } \\
\text { de palmas para cima. } \\
\text { O cachorro está ao } \\
\text { lado dele. }\end{array}$ \\
\hline $\begin{array}{l}\text { Pn4 } \\
\text { e } \\
\text { Pn5 }\end{array}$ & $\begin{array}{l}\text { CENA } \\
7\end{array}$ & $\begin{array}{l}\text { [a mãe aceita as desculpas/o pedido do menino } \\
\text { e /deixa que o cachorro ficar] }\end{array}$ & $\begin{array}{l}\text { Fazem /constroem uma casinha } \\
\text { Aceitável: } \\
\text { (fazem/constroem) } \\
\\
\text { (1 ponto) }\end{array}$ & $\begin{array}{l}\text { O menino, a mulher } \\
\text { (mãe) e o cachorro } \\
\text { estão juntos, ele tem } \\
\text { um martelo na mão, } \\
\text { ela tem um serrote ao } \\
\text { seu lado. Em frente } \\
\text { tem uma casinha de } \\
\text { cachorro. Há pregos } \\
\text { no chão. }\end{array}$ \\
\hline
\end{tabular}

Legenda: ( ) = possíveis substituições

[ ] =interpretação ou informação acessória

Nota: 12 proposições gerais 